Voix et Images

voixetimages

\title{
L'Écriture comme pratique fantasmatique. La Commensale de Gérard Bessette
}

Jean Fisette

Volume 1, numéro 3, avril 1976

Gérard Bessette

URI : https://id.erudit.org/iderudit/200035ar

DOI : https://doi.org/10.7202/200035ar

Aller au sommaire du numéro

Éditeur(s)

Les Presses de l'Université du Québec

ISSN

0318-9201 (imprimé)

1705-933X (numérique)

Découvrir la revue

Citer cet article

Fisette, J. (1976). L'Écriture comme pratique fantasmatique. La Commensale de Gérard Bessette. Voix et Images, 1(3), 329-337. https://doi.org/10.7202/200035ar d'utilisation que vous pouvez consulter en ligne.

https://apropos.erudit.org/fr/usagers/politique-dutilisation/ 


\section{L'Écriture comme pratique fantasmatique La Commensale de Gérard Bessette}

La récente parution de la Commensale ${ }^{1}$ nous rappelle un fait souvent oublié dans la critique: la double carrière d'écrivain et de critique de Gérard Bessette.

Le roman qui nous est proposé n'est pas une œuvre nouvelle; il s'agit d'un texte écrit entre le Libraire et l'Incubation. Cette parution tardive nous invite à une réflexion sur un discours un peu vieilli (dans son esprit) et à proposer quelques considérations sur la production de Gérard Bessette. Les interactions entre l'écriture du roman et la pratique critique (cette coupure est toujours moins importante que ne le suggèrent les étiquettes) invitent à voir dans la Commensale une image - plus ou moins fictive de l'herméneutique du langage.

Une simple considération d'ordre historique nous rappelle que Bessette est, à quelques années près, de la génération de Saint-Denys Garneau et de Paul-Émile Borduas. Quelle place prend-il alors dans l'évolution de la conscience québécoise?

On peut trouver un premier élément de réponse dans une récente étude de Jacques Blais sur la poésie québécoise du temps de la seconde guerre; au sujet de Saint-Denys Garneau, le critique propose cette observation très fine:

... la peur de s'illusionner, la distanciation d'avec le réel, le détachement intellectuel, le jeu littéraire lui-même aboutissaient à une ironie en sous-entendus, légère, à fleur de poème. Chez Garneau, plus profond et plus tragique, on aboutira à une ironie métaphysique? ${ }^{2}$.

Et il ajoute:

L'ironiste est emporté dans un mouvement circulaire vertigineux, un vagabondage chronique qui finit par le déréaliser lui-même, enjeu de son propre enjeu. Autant d'attitudes et de procédés bien familiers à Garneau ${ }^{3}$. 
Bien qu'il soit toujours périlleux de parler d'ironie à propos d'un texte, et que ce trait, chez Saint-Denys Garneau, soit très fin, peu perceptible à la première lecture, on admettra que cette observation, qui semble très juste, a le dcuble avantage de faire éclater les classifications traditionnelles ("les Aînés") et de permettre un renouvellement de la critique du poète, c'est-à-dire d'éliminer les thèmes mystificateurs du «poète maudit» et de placer Regards et jeux dans l'espace dans de nouvelles perspectives critiques (socio-historiques, psychanalytiques, etc.) et sur de nouveaux réseaux intertextuels.

Dans cette ironie, cette "peur de s'illusionner", ce "détachement intellectuel", on reconnaît la manière de Bessette dont tous les romans affichent, dans une parfaite concordance avec les ouvrages critiques, cette place prépondérante accordée au langage (mais ici non plus comme en "sous-entendus") provoquant un effet de "distanciation d'avec le réel». On pourrait proposer que le style, la préoccupation de Bessette représente la tentation impossible de Saint-Denys Garneau ${ }^{4}$, un prolongement et, vraisemblablement, un aboutissement.

En ce sens, G. Bessette serait; avec les esprits analytiques, du côté de Saint-Denys Garneau, contre les Automatistes, les Borduas, les PaulMarie Lapointe, ces intuitifs qui s'abandonnent totalement à la vie psychique; une grande "générosité » les anime comme s'ils voulaient embrasser totalement la vie, considérant le langage - peinture et écriture - comme translucide, comme l'instrument soumis et fidèle de l'inconscient. Dans le Vierge incendié, pour retenir le plus illustre des textes automatistes (accessibles), la parole est, non pas intense, mais, pourrait-on dire, schizophrénique: diverse, éparpillée, ambivalente; l'univers créé est atopique, utopique...

Chez Bessette, comme chez Saint-Denys Garneau, bien que dans des mesures extrêmement différentes, on trouve au contraire une propension, refoulée ou exploitée jusqu'au délire, à l'interprétation, fondée sur un scepticisme inaugural face aux choses (paranoïa d'interprétation).

Or, la Commensale met en scène ce mécanisme complexe d'interprétation, d'auto-évaluation qui nous semble coïncider avec la critique textuelle (à base interprétative, de plus en plus pratiquée aujourd'hui, et avec brio par $\mathrm{G}$. Bessette lui-même).

Réjean Robidoux affirmait:

La marque distinctive de Gérard Bessette ressortit en somme à la conscience de ce que l'aspect formel est le plus important en littérature ${ }^{5}$.

tandis qu'en réponse, Bessette dit: 
... le fond (la distinction me paraît légitime) est aussi important que la forme ${ }^{6}$.

II n'est pas dans notre projet de reprendre ce débat (sans fin), mais bien de proposer, au-delà des termes "fond/forme" un questionnement du texte sur son engendrement interne, montrant du fait, la vanité de cette question. (Compte tenu de la pratique critique de Gérard Bessette, on pourrait d'ailleurs se demander si ce débat - falsifié ? - n'a pas comme fonction d'obnubiler des préoccupations d'un autre ordre, et ceci aux dépens de l'interviewer.)

La Commensale, dans la perspective de la recherche formelle, - sur le plan du langage - va plus loin que le Libraire; par contre le vraisemblable référentiel (identification du narrateur à un personnage) est plus faible ou, tout simplement raté : de fait, cet appendice final qui met en scène Chayer rédigeant le livre qu'on vient de lire, non seulement n'est pas convaincant - alors que Jodoin, dans le Libraire, intégrait l'écriture dans ses activités - mais il est peu intéressant; loin de résoudre la question. du vraisemblable, ce rejet final pose ce problème que le lecteur n'aurait cure d'ignorer. Dans l'Incubation, cette question sera heureusement obnubilée: plutôt que d'un langage imaginaire intégré dans la fiction romanesque (le Libraire), on trouvera un langage imaginaire institué en ÉCRITURE, au sens d'un texte suffisamment puissant pour n'avoir pas besoin du vraisemblable romanesque pour se justifier. C'est qu'entre le Libraire et I'Incubatıon, le texte bessettien passe d'un état de langage proprement narratif à l'état d'écriture, non plus reflet (mimesis), mais production de sens (semiosis): le point d'appui du texte passe, de la référence (les choses, le monde sensible) à son propre engendrement: le point d'appui devient point de fuite.

La Commensale figure cette transformation, la permet, la réalise d'où le sentiment de malaise ressenti à la lecture. Texte problématique qui, précisément pour cette raison, invite à un questionnement sur l'écriture comme fantasme, le texte comme scène, identités non instituées, mais en devenir.

Entre Chayer et les autres figurants (Mme Bessière, Paulo, M. de Repentigny, et autres) - leur statut est indécidable: actants (comme sujets d'actions) ou personnages (comme entités psychologiques)? - il y a un écran: fine toile qui filtre la communication, les perceptions, l'affectivité, certes, mais aussi un écran de cinéma sur lequel se joue une "représentation " du monde que, semble-t-il, Chayer se donne à soi et laisse voir aux autres.

Mais, à la différence du journal de Jodoin, il ne semble pas que l'on puisse imputer à Chayer la paternité - responsabilité entière - de cette mise en scène: esquisse, écran, tableau noir du maître de mathématique ou de géométrie, certes, et l'écriture de la Commensale emprunte occasionnellement le ton didactique, l'allure démonstrative. II faudrait d'ailleurs remarquer que face aux figurants, Chayer réagit exactement à la façon d'un écolier face à ses maîtres à dénigrer (dessin, surnoms, etc.) Dans cette 
perspective du vraisemblable (rapport texte - référent), le texte est simplement caricatural, cynique et comique. Si, par contre, l'on considère le texte, non plus comme "représentation", mais comme scène, lieu de son propre enjeu, l'écriture apparaîtra comme lieu de manifestation, de la réalisation des fantasmes. Alors, la fonction de "représentation" sera refoulée, réduite aux additions et aux parties d'échecs mentales, au lexique plus ou moins régulièrement alimenté, diverses parties, jeux auxquels se prête Chayer pour meubler sa solitude, c'est-à-dire se représenter (se cacher/se dévoiler) lui-même à lui-même.

Ce qui conduit à la question suivante: quelle est la signification de l'opposition entre les valeurs incertaines (cahier de vocabulaire) et les valeurs sûres (chiffres et géométrie) ?

Les exercices chiffrés constituent un refuge, un point de repère (repaire), lieu des certitudes ne laissant aucune place à l'angoisse et où, second avantage, il y a place pour la compétition (évidemment avec soimême: abaisser son propre record); en regard de ces exercices, le cahier de vocabulaire fait figure de parent pauvre:

Je l'ai commencé voilà trente-deux ans et quatre mois, à une époque où je m'imaginais encore que les mots pouvaient avoir un sens précis. [...] Mais, durant les années qui suivirent (j'étais, rien moins que précoce), je me suis rendu compte que les uns et les autres mots [dénomination / définition] étaient d'une égale (ou quasi égale) insignifiance. Non seulement pour moi - ce qu'on aurait pu attribuer à la faiblesse de mon entendement - mais pour tout le monde. On n'a qu'à écouter parler les gens, à lire les journaux ou des livres pour être fixé la-dessus. (p. 34)

Par contre, le cahier sert aussi de réservoir; avant d'aller rencontrer $M$. de Repentigny pour la dernière fois, Chayer se gonfle à bloc dans son vocabulaire; si bien que le cahier, à la différence des exercices chiffrés, a une fonction présumée sociale; plutôt que de permettre un exercice narcissique, il est une "banque de données"; il est axé sur la vie sociale. Sous l'étiquette des valeurs certaines / incertaines, se profile, en fait, le couple solipsisme / altérité.

Les exercices chiffrés ont valeur d'autonomie. Et s'ils entrent en ligne de compte dans la description (assurant un vraisemblable romanesque), ils sont signes d'une fuite: c'est que les mesures induisent une représentation statique du flux du réel et, par conséquent une distanciation caricaturale (ironie): on n'a qu'à se rappeler la description-mesure, en termes de géométrie analytique, de l'omb.e sur le plancher d'Athanase, complètement abasourdi par l'alcool; mais, de ce fait, oublié; ce qu'on pourrait appeler: laisser la proie pour l'ombre. On n'est vraiment à son aise que dans la “représentation", car là on échafaude un jeu avec des unités pertinentes, limitées, graduées et métrisées (maîtrisées). Les mots ne peuvent être pure représentation: ils sont, à la fois instruments et objets d'analyse (d'où un processus dynamique), lieu de combat (logomachie) et de jeu (“logohédonie"). 
Or tout le texte de la Commensale représente un effort de systématiser le réel, le figer, c'est-à-dire de chiffrer les mots. C'est par cet aspect qu'à la différence du Libraire, on peut spécifier la dernière parution de Bessette; le flux textuel (on ne saurait encore parler du flux du réel) acquiert son autonomie par rapport à la psychologie et l'activité de Chayer.

Le texte qu'on a dénommé plus haut, écran, constitue une "scène" sur laquelle se conjuguent l'imaginaire et le raconté; une scène qu'on considèrera comme le topos des fantasmes:

Le fantasme est à la limite de la représentation et de sa cause. [...] il est précisément la limite même ${ }^{7}$.

De la même façon que l'objet perçu par l'œil n'est pas la chose ellemême, mais l'impact de la lumière sur celle-ci, l'écran textuel est à percevoir comme projection du regard de Chayer sur le monde; une projection / perception (le couple étant indissoluble) telle que Chayer procède à un échafaudage toujours plus complexe de façon à disparaître lui-même; et sa "disparition" sera assurée par un masque, le beau rôle qu'il se donne:

$\ldots$ je vous ai conçu [dit $M$. Bessière à Chayer] étonnamment semblable à ce que vous êtes: d'une intelligence au-dessus de la moyenne [...] oui, volontairement raccornie, désséchée - réduite au rôle, 1) de machine à calculer; 2) d'appareil à insulter les gens, à leur témoigner le mépris le plus absolu. C'est ce qui m'a inspiré le désir de vous connaître...

J'étais tout oreilles. II est toujours intéressant de se faire déboulonner avec méthode et précision... (p. 123)

Dans ce court fragment on trouve deux exemples, chez Bessière et chez Chayer, de ce double phénomène d'émission / perception et de leur rencontre. De fait, Chayer ne peut qu'être heureux de voir ainsi réussie (vraisemblable), l'image qu'il se fait et qu'il donne de lui-même; J.-B. Clément note exactement que «... le sujet qui fantasme, met en scène sa propre exclusion ${ }^{8}$ ".

Alors si le texte n'est pas «représentation» du monde sensible, ni projection adéquate de l'imaginaire chez Chayer, où le situer? Comment le saisir?

On pourrait, dans une première évaluation, naïve, le considérer comme "terrain de jeux", scène du plaisir (le combat y participant).

Ce plaisir est lié à la pulsion scopique; dans une perspective psychologique, on pourrait considérer la parole-action de Chayer comme la projection d'un regard cynique sur le monde et, en déduire un esprit sceptique. $\mathrm{Si}$, par contre, on s'en tient à une base d'analyse proprement psychanalytique, on considérera l'œil comme un appareil à la fois émetteur et récepteur, produisant un circuit d'ondes visuelles qui, à la façon d'un boomerang, revient au sujet.

II n'est que de retenir ces longues descriptions du faciès des figurants; la tête de Passetout, par exemple:

... bien que le bourrelet adipeux (ou le pneumatique) qui occupe le deuxième rang de bas en haut, c'est à dire en partant du menton 
proprement facial, écrase de sa masse celui qui le précède (le menton proprement dit) et les deux qui le suivent, à savoir: le deuxième bourrelet médian et le bourrelet terminal férocement étranglé dans sa partie inférieure par le celluloïde du col ecclésiastique, il faut tenir compte des pneus secondaires aussi bien que du principal.

On en arrive ainsi, au point de vue topographique, au nombre quatre. D'autre part, si on se place au simple point de vue masse, on aboutit à un chiffre approximatif (puisque jaugé à vue de nez) de 1,333 , les bourrelets secondaires ne représentent en effet respectivement qu'un sixième, un vingt-quatrième, un huitième de la masse apparente du bourrelet principal. (p. 76)

sera détaillée sur une base topographique (crevasse est-ouest du double menton) et chimique (masse, à vue de nez, de 1,333). Le trajet effectué par le regard, loin de se fixer sur l'objet (c'est le paradoxe de la métaphore scientifique) s'en retourne à son point de départ, s'enliser dans des catégories (cadres de mesure) et, par conséquent, boucler la boucle. La circularité est assurée aux dépens de l'objet en soi, qui demeure pratiquement étranger au circuit, remplacé qu'il est par les mots. Dès lors, le texte apparaît comme flux, cheminement circulaire des ondes visuelles, point de jonction ("collapsus") entre l'émission et la réception du circuit visuel.

Si l'on retient la définition que propose Serge Leclaire du plaisir,

... expérience immédiate (perception) d'une différence, d'une rupture produite par l'achèvement d'un circuit pulsionnel élémentaire ${ }^{9}$.

on verra du côté du lecteur - qui produit lui aussi son circuit scopique: la pratique de la lecture - la lettre, le texte engendrer le plaisir par la réduction de l'écart (DIFFÉRENCE) entre la réalité connue et sa « représentation »; d'où le comique...

Or Chayer est incapable de plaisir, d'amusement, sinon cynique; c'est que les mots, le texte, chez lui, ne remplissent pas, d'une façon relativement autonome, cette fonction d' «anti-corps" à la DIFFÉRENCE; les mots, la lettre lui collent à la peau, font corps avec lui. C'est lui-même, en tant que dominé par la pulsion scopique, qui est, dans cette scène fantasmatique, pris à partie, impliqué.

Le désir, chez Chayer, analysé en termes de déplacement, peut se lire précisément dans cette projection qu'il fait de lui-même sur l'autre; mais paradoxalement le texte-écran cache l'autre pour le transformer en un miroir du moi; la projection du désir se transforme en narcissisme qui, ne pouvant et/ou ne voulant s'avouer, se déguise sous l'image rassurante socialement (sur-moi) de la rigueur mathématique.

Si bien que la métaphore scientifique, en termes psychanalytiques, serait à percevoir comme une substitution qui a comme fonction d'ajouter un "plus" au réel, tandis que la parole de Chayer, réalisant une condensation des choses et du regard, soustrait un "moins" au réel.

Chayer figure, pourrait-on dire, l'apprenti-sorcier de la lettre, à laquelle il est "gommé"; le phénomène essentiel à remarquer est celui de l'abolition de la distance... du plaisir. 
Lorsque nous disons que, dans la psychose, quelque chose d'essentiel à la fonction de la lettre défaille, c'est pour signifier que l' "anticorps " qui doit marquer la différence n'est pas produit comme tel. Or, seul cet anti-corps, à savoir la lettre ou le mot, permet que s'instaure l'ordre du plaisir (Lust) dans une altérité radicale par rapport au corps biologique, c'est à dire dans l'inconscient. Dans la psychose, tout se passe comme si cette fonction AUTRE de la lettre était récupérée au niveau du corps, annulant le clivage de l'altérité elle-même ${ }^{10}$.

Ce qui, au dire de Serge Leclaire, caractérise le psychotique, incapable de plaisir.

Le texte, malgré sa prétention et son allure d'objectivité (désir exprimé chez Chayer par la métaphore du chiffre, effort de "représentation" de lui-même), ne réussit pas à atteindre le monde sensible. Ce mécanisme, par lequel les choses, les événements ne sont jamais investis, mais demeurent toujours comme frôlés, commande tout le texte. II n'est que d'examiner un autre fragment relatif au «traitement d des événements:

Là-dessus, Madame Bessière a poussé un profond soupir ; elle a tiré son mouchoir de sun sac et elle s'est mouchée. Quand j'ai vu 'qu'elle ne disait rien, je me suis remis à manger. Elle a replacé son mouchoir dans son sac, a pris sa fourchette et son couteau, a découpé quelques tranches de canard. Mais elle ne faisait que chipoter. Je me suis dit que je n'avais sans doute pas abordé le problème de la bonne façon et qu'il me faudrait revenir à l'attaque une autre fois. Je 'm'étais trompé. Madame Bessière a soudain déposé ses ustensiles dans son assiette pour déclarer que si elle gardait le silence c'est qu'elle avait trop de choses à dire. Elle ne savait par où commencer. (p. 24 ; c'est nous qui soulignons)

Bien que l'emploi du style indirect, qui fusionne reportage et commentaires, passés ou présents, rende le phénomène moins explicite, on peut aisément relever la superposition de deux niveaux de parole chez Chayer: événements oraux actualisés (fin du paragraphe) et réflexions (événements oraux non actualisés: passage souligné). Ces deux niveaux relevés sont en constante relation d'interdépendance, c'est-à-dire que la parole actualisée de Chayer n'est jamais libre, mais au contraire, toujours soumise à un calcul, prospectif ou rétrospectif; elle est toujours chiffrée, sur le modèle de "représentation" des exercices échiquiers mentaux; à la différence près que les mots, en plus de n'être jamais absolument certains, ne permettent pas de distanciation par rapport au sujet, interdisant, de ce fait, le contentement, le plaisir.

Si nous avons ainsi considéré Chayer comme une personne (entité analysable en termes d'émission / réception d'une pulsion scopique), c'était pour répondre à une nécessité / fiction théorique: celle de fonder l'analyse. Pour répondre aux caractéristiques du fait littéraire en tant qu'écrit, on peut maintenant inverser les perspectives.

En effet, Chayer n'a pas, à proprement parler, de substance, d'ordre psychologique ou psychanalytique, qui revendiquerait le texte; et l'on ne sera pas dupe du rejet final le décrivant dans un travail quelconque de rédaction. 
Ce qui s'impose en premier lieu, c'est le texte qui, en vertu de son étagement continuel, se justifie, s'engendre constamment. Tout repose sur ce mécanisme d'auto-commentaire et d'auto-évaluation qui confine, nous l'avons souligné plus haut, à une structure délirante apparentée à la paranoïa d'interprétation, de mesure, de classification (arithmomanie). Ainsi, c'est l'épaisseur textuelle qu'il faudrait décrire en termes psychanalytiques. Chayer, comme les autres figurants d'ailleurs, n'y figure que comme un effet décalé, un sous-produit qui ne prend de signification qu'au niveau du dogmatique vraisemblable romanesque. C'est, semble-t-il ce qu'a compris G. Bessette lorsqu'il entreprend l'Incubation où le langage défilera d'une façon autonome, c'est-à-dire débarassé du paravent encombrant qu'est le personnage-narrateur.

On comprendra alors que le débat suggéré plus haut, à propos de prédominance entre fond et forme n'a plus, à proprement parler, aucun sens. Il serait de même vain de tenter de réduire les exercices mentaux à la simple catégorie d'une "métaphore du chiffre»: ce serait ne rien dire; plutôt que de simple écho, effet décalé, il faudrait considérer ce leitmotiv comme remplissant une fonction de "représentation " en abime, «sur-moi " de la pratique d'inquisition du monde, des choses, manifestant une propension refoulée au narcissisme: le texte reste prisonnier de cette dialectique insoluble du voilement / dévoilement: de fait, ce narcissisme constitue l'élément qui fonde la dynamique même du texte.

On ne peut, suivant la proposition de Jacques Derrida, que «... faire sauter l'opposition rassurante du métaphorique et du propre dans laquelle l'un et l'autre ne faisaient jamais que se réfléchir et se renvoyer leur rayonnement ${ }^{11}$ ". Cette "représentation " en abîme génère la scène par une triple fonction: engendrer, réaliser et limiter à la fois le fantasme de la pulsion scopique. Si bien que la "scène" proprement dite serait à situer dans un espace, un interstice, impossible à localiser exactement, entre la parole de Chayer et ses exercices mentaux, deux pratiques qui manifestent un effort contradictoire de voilement / dévoilement.

Il devient difficile de soutenir la thèse ironiste à propos de Bessette; de fait, il fait éclater cette forme de "méfiance" qui définissait Saint-Denys Garneau; les ouvrages de G. Bessette (création et critique) posent franchement la question de l'herméneutique du langage: question qui, aujourd'hui, devient peut-être l'obsession caractéristique de notre imaginaire intellectuel. 
1. G. Bessette, /a Commensale, roman, Montréal, AS/Quinze, 1975.

2. J. Blais, De l'ordre et de l'aventure. La Poésie au Québec de 1934 à 1944, Québec, PUL, 1975, "Vie des lettres québécoises", $n^{\circ} 14$, p. 147.

3. lbid., p. 148.

4. Si l'on se reporte à l'excellente étude de $\mathrm{J}$. Blais sur la signification des figures géométriques dans l'imagination garnienne, on verra une première manifestation de l'“arithmomanie»: Saint-Denys Garneau et le mythe d'lcare, Sherbrooke, Cosmos, 1972.

5. Réjean Robidoux, "le Cycle créateur de Gérard Bessette ou le fond c'est la forme", dans Livres et auteurs québécois 1971, Montréal, Jumonville, 1972, p. 11.

6. "Bessette par lui-même", entrevue dans le Québec littéraire 1, Montréal, Guérin, 1974, p. 140.

7. C.-B. Clément, "De la méconnaissance: fantasme, texte, scène" dans Langages 31, Paris, Didier-Larousse, septembre 1973, p. 37.

8. Ibid., p. 44.

9. Serge Leclaire, “les Mots du psychotique" dans Change: Déraison/Désir, $n^{\circ}$ 12, Paris, Seuil, septembre 1972, p. 123.

10. Ibid., p. 129.

11. J. Derrida, "la Mythologie blanche (la métaphore dans le texte philosophique)", dans Poétique, $n^{\circ} 5$, Paris, Seuil, 1971, p. 52. 